\title{
PARTIII.
}

\section{MEDICAL MISCELLANY}

\section{Reports, Transactions, and Scientific Intelligence.}

Eczema. By M. P. Aubert. Translated by George M. For, M.D., F.R.C.S.I. ; Surgeon to the Whitworth Hospital, Drumcondra.

M. P. Aubert (in the Lyon Médical) writes:-Meteorologists, a prudent race, inspired by the true scientific spirit and tanght of experience, when they have consulted their carefully-kept registers, issue their weather prognostications u nder the word "probably," and then follows the usual intimation "fine," or " wet," as the case may be. Dermatologists, who base their deductions on clinical experience, or, better still, on the mi croscopic characters of their pathological specimens, might with benefit follow the example of the meteorologists, and prefix "probably" when writing of eczema and many other skin diseases. When we see a patch of eczema with a more or less irregular surface, red and moist, exposed to contact with all sorts of substances, in no point is it in touch with the spirit of science to assert that parasites, which may be present, are the originating cause of the disease, or that they are speeific, and in no sense a complication. The question of the microbic origin of eczema has for a long time occupied the attention of dermatologists, and sufficient progress has been made in the study to justify the order of the day, issued at the late International Congress of Medicine, calling for a series of reports on the subject. Unna, of Hamburg, has no doubt that eczema is a contagious malady, even sometimes occurring as an epidemic. The many varieties of its form are, he thinks, due to the many species of microbes that take part in its production. To determine the species and make plain their characters is the best way to arrive at a proper solution of the question. Jadarsolin, of Berne, admits that on the efflorescence of the disease he failed to find the micro-organisms, but after a little time he obtained germs which, being dereloped, manifested pathological activity. Galloway, of London, thinks many factors play a part in the production of the disease; chief among these he places microbes. To him it appears that there must be a condition of the skin predisposing to the disease, a general state of ill-health, in which digestive troubles play an. 
important part. Broeq and Veillon, of Paris, dwell on the fact that the initial vesicle does not contain any microbe detectable either by the microscope or by cultures. The abundant crop of microbes and their great diversity in form, which are found later on, are due to adventitious causes, and have nothing to do with causing the disease. In the discussion which followed, in which Brocq, Kaposi, Unna, Andry, Sabourand, and others took part, all the statements of Sabourand, based on numerous microscopic preparations, and which go to show that the initial lesion of eczema is free of micro-organisms, although the vesicle of impetigo always contains them, were re-told. Morgan Dockrell, by the aid of photographic preparations, demonstrated the impossibility of sustaining the theory that eczema is a parasitic disease. The discussion continued without adding anything of importance to what we have stated. With all this what conclusion are we to arrive at? Without doubt we should imitate the eaution of the meteorologist. On one point the medical world is of one opinion-namely, that eczema is not an entity, but a group of divers affections. On another point the French School of Medicine is unanimous - to wit, that microbes, when present, are due to adrentitious circumstances. Lesions, in which microbes are present from the beginning, and are the cause of the disease, may closely resemble eczema, but they are not true eczema. According to the French school eczema is not a microbic disease, neither is it infectious. It is due to an external cause acting on a skin predisposed to the disease. What is the cause? The question was not considered at the Congress, but the last number of the Annales de Dermatologie contains a long article by Professor Tomnasoli, of Parma, who believes he sees dermatologists going astray after micrococci and staphylococci, insists energetically on the alloxuric theory of eczema, and we recall the rejuvenated theories of arthritis.

POPliteal ANEURYSM CURED BY Digital COMPRESSION OF THE. FEMORAL.

Dr. Hamann (Cleceland Journal of Medicinc) gives the case of a negro, aged forty-two, who had an aneurysm situated on the popliteal artery about the size of a hen egg. The treatment was digital compression, kept up for thirty-six hours by relays of medical students. A splint was applied for three or four days, and in a week the patient was allowed to get up.

[The case recalls the many similarly successful results obtained by digital compression in Dublin in the first half of the last century.] 